\title{
Tree nut allergy
}

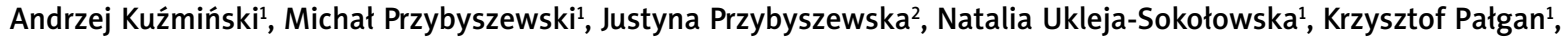 \\ Zbigniew Bartuzi ${ }^{1}$
}

\author{
${ }^{1}$ Department of Allergology, Clinical Immunology and Internal Diseases, Collegium Medicum in Bydgoszcz, Nicolaus Copernicus \\ University in Torun, Poland \\ ${ }^{2}$ Department of Nutrition and Dietetics, Collegium Medicum in Bydgoszcz, Nicolaus Copernicus University in Torun, Poland \\ Adv Dermatol Allergol 2021; XXXVIII (4): 544-549 \\ DOI: https://doi.org/10.5114/ada.2021.108894
}

\begin{abstract}
From a botanical point of view, a nut is a kind of dry, closed, unbroken, single-seeded fruit, which has a ligneous pericarp made up of several fruiting bodies surrounded by one free semen. The term "allergy to tree nuts" includes allergies to almonds, Brazil nuts, cashew nuts, hazelnuts, chestnuts, macadamia nuts, pecan nuts, pistachios and walnuts. All tree nuts belong to five orders of plants: Rosaceae, Fagales, Sapindales, Ericales and Proteales. Allergies to tree nuts usually start in childhood, but unlike other food allergies, they rarely fade away and generally persist throughout life. They are one of the main causes of serious allergic reactions ending in death in both children and adults. The epidemiology of allergy to nuts is variable and depends mainly on the geographical zone and eating habits. In northern Europe, hazelnut allergy is prevalent, whereas in the USA the most common is allergy to peanuts and, when taking tree nuts into consideration, to walnuts. The diagnosis of allergies to nuts is difficult and requires the use of the most modern research tools including molecular diagnostic techniques.
\end{abstract}

Key words: tree nut allergy.

\section{Introduction}

From a botanical point of view, a nut is a kind of dry, closed, unbroken, single-seeded fruit, which has a ligneous pericarp made up of several fruiting bodies surrounded by one free semen. The pericarp of the nuts is not grown with the semen and falls away from the mother plant entirely. Nuts are also commonly, albeit wrongly, called the edible seeds of many different plant species, the fruits of which are in fact bags (e.g. Brazil nuts picked from the lofty nut), stone fruits (e.g. walnut, trifoliate macadamia, coconut proper), pods (e.g. so called groundnuts) or Pini seeds [1].

The term "allergy to tree nuts" includes allergies to almonds (Almond), Brazil nuts (Brazil), cashew nuts (Cashew), hazelnuts (Hazelnut), chestnuts (Chestnut), macadamia nuts (Macadamia), pecan nuts (Pecan), pistachios (Pistachio) and walnuts (Walnut). All tree nuts belong to five orders of plants: beech (Fagales), soapwood (Sapindales), silverberry (Proteales), heather (Ericales) and rosewood (Rosales). Among the beech trees, there are chestnuts belonging to the beech family, nut and walnut belonging to the Juglandales and hazelnut (birch family). Silverberries are represented by macadamia nuts, which belong to the silverberry family. In turn, pistachio are representatives of the Sapindales order. he Brazil nut family, the Lecythidaceae, is in the order Ericales, while the almond belongs to the Rosaceae family, of the Rosales order [2].

Allergies to tree nuts usually start in childhood, but unlike other food allergies, they rarely fade away and generally persist throughout life. They are one of the main causes of serious allergic reactions ending in death in both children and adults [3]. The frequency of allergies to tree nuts increases and the main reason for this is that they are a frequent snack and also often occur as so-called latent allergens. The Food Allergy and Anaphylaxis Network (FAAN), after analysing data of 5149 people (USA), reported that among tree nut allergies, walnuts (34\%), cashew nuts (20\%), almonds (15\%), pecan nuts (9\%) and pistachio nuts (7\%) were the most common causes; less frequently, hazelnuts, Brazil nuts and macadamia nuts (below $5 \%$ in each case) [4].

Address for correspondence: Andrzej Kuźmiński MD, Department of Allergology, Clinical Immunology and Internal Diseases, Collegium Medicum, Nicolaus Copernicus University, 75 Ujejskiego St, 85-168 Bydgoszcz, Poland, phone/fax: +48 523655 416, e-mail: przybysznr1@interia.pl

Received: 13.11.2020, accepted: 3.03.2021. 


\section{Common almond, plum almond (Prunus dulcis)}

The almond is a fruit of a shrub or a small deciduous tree belonging to the Rosaceae family. It grows best in areas with dry and warm climates and comes from North Africa and the Middle East - it grows wildly in Sicily and Greece and is widely cultivated in northern Africa, southern Europe, Australia and the warmer regions of the United States, especially in California. Almonds come in many varieties, two of which are best known: sweet and bitter. Externally they are similar, but differ in their chemical composition - bitter almonds contain significant amounts of amygdalin, which contain hydrocyanic acid [1].

A number of allergens of different molecular weights were isolated from almonds: 12, 30, 37, 45, 50 and 62 kDa. Some of these proteins were found to be similar to $7 \mathrm{~S}$ globules and $2 S$ albumin [5]. Two proteins were described as the main allergens, one of which is thermolabile and the other thermally stable. The main allergen of the almond, responsible for most allergic reactions, is the storage protein (AMP, amandine), which is a highly thermolabile protein and is probably equivalent to the previously described $2 S$ albumin (belonging to the prolamin family) [6].

So far, the following allergens of almonds have been characterized:

- Pru du 1 (2S albumin, spare protein);

- Pru du 2 (conglutin, 7S globulin, reserve protein);

- Pru du 3 (LPT, lipid transporting protein);

- Pru du 4 (profilin);

- Pru du 5 (ribosomal protein P2);

- Pru du 6 (amandine - hexameric protein of $360 \mathrm{kDa}-$ 11S legumin) [7].

Amandine is a leguminous protein, is not a glycoprotein and consists of 2 major polypeptides with a molecular weight of 42-46 and 20-22 kDa, connected by disulphide bonds [7].

The main allergen of almonds, $2 \mathrm{~S}$ albumin, leads to cross-reactions with other foods containing this protein: walnuts, sunflower seeds and peanuts, among others $[5,6]$. Pru du 2, like Ara h 2 - the main peanut allergen, is conglutin. In patients with peanut allergy with the presence of antibodies directed against Ara h 2, crossreactions with allergens of Brazil nuts and almonds have been shown [8]. Pru du 3 (LTP) almonds have a sequence identical to $94 \%$ of the LTP of apricots [9]. For Pru du 3, $62 \%$ of the amino acid homologation with LTP of the hazelnut and $59 \%$ each with the peach and cherry was shown. Pru du 4 shows cross-reactivity with other plant profiles (however, this protein is denatured, which causes its variable reactivity) [10].

The relationship between pollen allergy and allergy to almonds was also described: as much as 33\% of birch pollen allergy patients had a simultaneous hypersensitivity to almonds [11].

\section{Peanut trees, Brazilian Peanut trees, Bertholletia excelsa}

It is an evergreen tree, from the Brazil nut family, the Lecythidaceae, in the order Ericales. In the wild it occurs in the tropical forests of the upper Amazon basin: mainly in Bolivia, but also in the western and northern states of Brazil and Peru, and a few in Colombia, Guyana, Suriname and Venezuela [1]. Brazil nut proteins, although they constitute only $15-17 \%$ of the fresh weight of nuts, constitute as much as $50 \%$ of the defatted flour. The most important allergens are spare proteins: $11 \mathrm{~S}$ legumin and $2 \mathrm{~S}$ albumin [12].

So far, the following components have been characterized: Ber e 1 (2S albumin); Ber e 2 (11S globulin).

Ber e 1 is associated with the occurrence of serious allergic reactions. It occurs in large quantities in Brazil nuts and is resistant to temperature and digestive processes [13]. $2 \mathrm{~S}$ albumin is similar to $2 \mathrm{~S}$ albumin of cotton, cocoa beans, sunflower seeds, rapeseed, walnut (Jug r 1), mustard seeds (Sin a 1) and sesame seeds (Ses i 2). The comparison of the amino acid sequence $2 \mathrm{~S}$ albumin of Brazil nuts and sunflower seeds showed as much as 34\% similarity and the similarity of Ber e 1 to 25 albumin of other plants is $38-52 \%$. Jug $r 1$ shows an identicality in $46.1 \%$ to Ber e 1 [14]. 2 S albumin plays an important role in the cross-reaction between walnut (Jug r 1 ) and pecan (Car i 1). Conformational analysis of linear IgE binding epitopes mapped on the surface of the Ara h 2 and Ber e 1 molecule did not show structural homology, which suggests that the observed cross-reactions between peanuts and Brazil nuts may depend on other protein panallergens, e.g. vicilin [15].

In patients with walnut anaphylaxis, the crossreaction with hazelnuts and Brazil nuts was described [16]. A British report from the Isle of Wight (population $125,000)$ described 12 cases of allergy to Brazil nuts between 1983 and 1991: 11 patients developed angioedema, 7 generalized urticaria, 5 bronchospasm, 2 stridor, 2 compressions in the throat, 2 itchy lips and 1 fainting. The onset of symptoms was less than 1-3 min after consumption of Brazil nuts [17].

\section{Western cashew (Anacardium occidentale), adrenal nut, cashew}

It is a tree species from the family of nano-chewing, a close relative of mango. It comes from the tropical areas of South America. It is grown on a large scale in most tropical countries, mainly on the southwest coast of India, in Vietnam, Nigeria, Mozambique, Tanzania and Brazil [1].

Dietary allergies to cashew are associated with the presence of IgE antibodies directed against the main backup proteins: $11 S$ globulins (a group of legumes) and $2 S$ albumin, which are also the main allergens in various plant seeds. $11 \mathrm{~S}$ globulins have a molecular weight in 
the range $31-35 \mathrm{kDa}$ and $2 \mathrm{~S}$ albumin are low molecular weight proteins [18].

So far, the following allergenic cashews have been characterized: Ana o 1 (7S globulin, vicilin); Ana o 2 (11S globulin, legumin); Ana o 3 (2S albumin); Ana o 4 (Profilin).

Ana o 4 is not clinically relevant. Epitopes of Ana o 1 are not linearly similar to peanut vicilin. Recombinant Ana 01 binds $50 \%$ of the sera of patients with a cashew nut allergy and $25 \%$ of patients with an allergy to other nuts $[18,19]$.

The Ana o 3 reserve protein is associated with the occurrence of severe allergic reactions and is found in large quantities in cashew nuts. It is resistant to temperature and digestive processes $[19,20]$.

High cross-reactivity between cashews and pistachios was demonstrated. Ana o 2 shows great similarity with walnut legume, which suggests cross-reactivity [19]. Recent studies have shown a significant homologation between 11s globulin (Jug r 4) of the walnut and the cashew (Ana o 2) and hazelnut (Cor a 9) [21]. It has also been shown that peanut (Ara h 1), walnut (Jug r 2), hazelnut (Cor a 11) and cashew (Ana o 1) have a similar structure, which suggests the possibility of cross reactions [21, 22].

\section{Common hazel (Corylus avellana)}

It is a large, multi-trunk bush belonging to the birch family. It occurs in the wild throughout Europe and Asia, especially in the region of South Caucasus. In Poland the common hazel grows both on lowlands and in mountains up to about $1300 \mathrm{~m}$ above sea level [1].

So far, the following hazel allergens have been characterized: Cor a 1 (protein PR-10); Cor a 2 (profilin); Cor a 6 (isoflavone reductase approval); Cor a 8 (non-specific LTP type 1); Cor a 9 (11S legumin, storage protein); Cor (LBP, luminal binding protein); Cor a 11 (7S vicilin, storage protein); Cor a 12 (oleosin of 17 kDa molecular weight); Cor a 13 (oleosin of molecular weight 14-16 kDa); Cor a 14 (2S albumin).

Among the hazelnut components, thermal shock proteins were also isolated [23].

Cor a 1 is a thermolabile protein sensitive to digestive processes, so heat-treated hazelnuts are well tolerated. Allergy related to this protein is mainly associated with local reactions [24]. Cor a 1 is responsible for cross reactions with pollen from birch trees and shows $80.5-83 \%$ homologation to Bet v 1 (birch), 83.6-85\% to Aln g 1 (alder) and 89.3-95\% to Car b 1 (hornbeam) [25]. Another component responsible for cross-reactions between birch pollen and hazelnut may be Cor a 2 (profilin), but the clinical significance of this reaction has not yet been proven [26]. Cor a 8 is resistant to high temperature and digestive processes and the allergy associated with it concerns both local and systemic reactions. Cor a 8 is responsible for cross reactions with LTP of other plants, i.e. peaches, peanuts, walnuts and cherries [27]. Cor a 9 and Cor a 14 are present in the hazelnut in large quantities and are resistant to high temperature and digestive processes. Allergy to them is mainly associated with systemic reactions. Cor a 9 (11S globulin) shows 45-50\% homology with peanuts and soya proteins [28].

\section{Edible chestnut (Castanea sativa, chestnut)}

It is a tall tree, once a species found only south of the Alps, spread by the Romans in Western Europe and Slovakia. It is now widespread in the Mediterranean, Asia Minor and Caucasus [1].

Chestnut is considered to be the 3rd most common food allergen in adults.

So far, the following chestnut allergens have been characterized: Cas S 1 (PR-10, Bet v 1 homolog); Cas s 5 (chitinase); Cas s 8 (non-specific LTP 1); Cas s 9 (small thermal shock protein class I).

In Korea, chestnut is considered to be the $3^{\text {rd }}$ food most common food allergen in adults.

Cas $\mathrm{s} 1$ is not considered a food allergen due to its high sensitivity to temperature and digestion. Cas $s 5$ belongs to the chitinases, the main fruit panallergens associated with latex fruit syndrome. Cas 58 is considered to be a very strong allergen. Cas $s 9$ is a chestnut allergen with the best known amino acid structure, with a strong conservatism in structure [29].

Cas $s 5$ and Cas $s 8$ are considered to be the main allergens, therefore some authors suggest that chitinase and LTP can be used as an excellent diagnostic tool in people allergic to chestnuts. An allergy to chestnut is rarely isolated, and is mainly associated with latex vesicles and food. After avocado and banana, chestnut is the third most frequent food associated with this syndrome. In a study of 22 people with a confirmed allergy to chestnut (in DBPCFC) at the age of 5 to 70 years, the chestnut spot tests were positive in $71 \%$ and slgE was detected in $54 \%$. Moreover, $86 \%$ of patients were allergic to plant pollen and $59 \%$ to Latex [30].

In a study by Lee et al. in 2005 evaluating the clinical relevance of chestnuts as a food allergen (1738 patients with ANN), skin reactivity to chestnuts was found in 56 (3.2\%) [31]. An interesting case of OAS caused by chewing chestnut by a 22-year-old woman is described. While chewing chestnut, she felt burning and itching of the oral and pharyngeal mucosa, followed by lacrimation, nasal itching, sneezing, and "moderate" dyspnoea. The commercial skin test, prick-by-prick test with fresh chestnut and slgE were negative, only DBPCFC was positive [32].

\section{Macadamia (Macadamia)}

It is a kind of evergreen trees found in the wild in Australia, New Caledonia and Sulawesi. The largest producer is Australia, but they are now also grown in Hawaii and South Africa [1]. 
So far, macadamia nut allergens have not been characterized. It seems that the main allergen can be a protein of $17.4 \mathrm{kDa}$ present in extracts from raw and roasted nuts. A protein with antibacterial properties from the Vicilin group has also been isolated, but its allergenicity has not been determined [33].

For reasons unknown so far macadamia nuts are poisonous to dogs. Symptoms may occur already after administration of $2.4 \mathrm{~g}$ per kilogram of body weight (a dozen or so shelled nuts for a dog weighing $10 \mathrm{~kg}$ [34].

A partial cross-reactivity between macadamia and hazelnut was shown. Thanks to the Western Blot method, antibodies reacting with proteins of extracts produced from walnuts, Brazil nuts, cashew, hazelnuts, macadamia, pistachio and sesame seeds were found in the serum of almond allergic patients [35].

An American study of 115 patients aged $4-19.5$ years described allergic reactions to macadamia nuts in $13 \%$ and $4 \%$ of the patients with moderate to severe symptoms.

In a survey (telephone), out of 118 patients reporting allergies to peanuts or tree nuts, only 2 patients reported allergies to macadamia nuts. A case of a 36-year-old patient was also described in which 5 min after eating chocolate with macadamia nuts, swelling of the back of the tongue, dysphagia, pressure and pain in the chest and skin itching appeared. Skin tests with fresh nuts were positive $[36,37]$.

\section{Edible nut, pecan nut, fruit nut (Carya illinoinensis), Pecan}

It is a long-lived deciduous tree with a height of 20$40 \mathrm{~m}$, found in warmer regions of North America, mainly in the USA along the lower Mississippi. The Spaniards gradually introduced this species to southern Europe and then to Africa and Asia. It is rare in Poland, mainly due to its high sensitivity to frost [1].

So far, the following allergens have been characterized: Car i 1 (2S albumin, storage protein), Car i 4 (11Sglobulin, legumin, storage protein).

The profile of the allergenic components in pecan nuts can vary with the ripening of the tree, but also with the storage and heat treatment of the nuts. The presence of proteins resistant to high temperature and digestive processes associated with severe systemic reactions was also demonstrated [38].

Clinical observations confirm the high cross-reactivity of the edible peanut with other peanuts (walnuts) and less with peanuts. The main walnut allergen Jug $r 1$ shows a high structural homology with Car i 1. Analysis of the linear and conformational epitopes of the Ara h 2 component showed no significant structural homology with walnut (Jug r 1), pecan (Car i 1) and Brazil nut (Ber e 1) components [15, 39].

Peanut allergy often appears in childhood and does not go away with age. The Food Allergy and Anaphylaxis
Network register (FAAN), which includes 5149 tree nuts allergy sufferers, estimates the frequency of allergies to peanuts at $9 \%$. Protein contact eczema to pecans was also described [36, 40].

\section{Pistachio (Pistacia vera)}

It is a dioecious shrub or a tree with evergreen or seasonal leaves up to $24 \mathrm{~m}$ high, growing in the northern hemisphere in warm or moderate climate. The fruit is small and often fleshy drupes, gathered in clusters [1].

So far, the following allergens have been character-

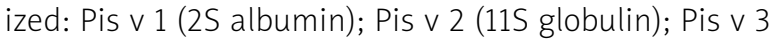
(vicilin); Pis v 4 (peroxide dismutase) [41].

Some studies also suggest the occurrence of LTP proteins characteristic for the whole order of soaps [42].

Rance et al. found that in 42 children allergic to cashew nuts, 7 showed simultaneous pistachio allergy. It suggest cross reactions between pistachios and cashew nuts [43]. The cross-reactivity of $11 S$ globulin (Pis v 2) and vicilin-like (Pis $\vee 3$ ) proteins with foods containing these allergens is possible, but has not yet been described [44].

It is estimated that allergy to pistachios is quite rare. Allergic symptoms of the oral cavity, skin, angioedema and severe anaphylaxis are observed [45]. Liccardi et al. described 3 cases of simultaneous anaphylaxis to mango and pistachio: a 3-year-old boy with facial oedema and stridor in a few minutes after pistachio ice cream; a 28-year-old woman with generalized urticaria after eating peppers, curry and celery and 3 months later itching of the mouth, face and tongue and nausea and a 28-year-old man with generalized itching and urticaria, abundant sweating, abdominal pain, nausea and vomiting immediately after eating peanuts, peaches, peppers, hazelnuts or mangoes. All these patients had a positive skin test with pistachio and fresh mango [46].

\section{Walnut (Juglans regia)}

It is a long-lived deciduous tree $25-35 \mathrm{~m}$ high. It belongs to light and thermophilic species. It grows and yields best on fertile, warm, rich in humus and calcium, alkaline soils (loess, black earth, brown soils and clay). In the wild it can be found in the Balkans, south-eastern Europe, south-western, central and eastern Asia, the Himalayas, northern Myanmar and south-western China. In Poland it is commonly cultivated and often wild [1].

So far, the following allergens have been characterized: Jug r 1 (2S albumin, spare protein); Jug r 2 (7S vicilin, reserve protein); Jug r 3 (non-specific lipid transporting protein (nsLTP)); Jug r 4 (11S globulin, spare protein).

Jug $r 1$ (main allergen) is detected in blood serum in up to $75 \%$ of patients with a walnut allergy (USA). An Italian study conducted in 46 OAS patients confirmed by an open provocation test showed that the main allergen detected was Jug r 3 (78.2\% of patients), while $21.7 \%$ of allergy suf- 
ferers identified proteins from the vicilin group. Allergens Jug $r 1$ and Jug $r 2$ are also representative for other nuts (black, grey, California, Manchurian, Siebolda) [47, 48].

Many studies have highlighted the high 2S-dependent albumin and other protein reserves, the cross-reactivity between walnuts and pecans, as well as almonds, hazelnuts, Brazil nuts, cashews, pistachios, peanuts and 2Sdependent albumin and vicilin between walnuts and sesame seeds. In people allergic to the main peanut allergen (Ara h 1), it is recommended to avoid the walnut (Jug r 2), the hazelnut (Cor a 11) and the cashew (Ana o 1). The lemons of peanuts (Ara h 3), walnut (Jug r 4), hazelnut (Cor a 9) and cashew (Ana o 2) have high structural similarity [49].

In 1/3 of patients with a peanut allergy, an allergy to tree nuts co-occurs. By examining 20 patients with LTP-dependent food allergy to rosaceous fruits (apples, pears, peaches, cherries, apricots, plums, or almonds) in as many as $80 \%$ of them Asero et al. showed clinical reactivity to nuts (hazelnut, hair and pistachio): in 11 (55\%) cases nuts caused systemic reactions, in 9 cases urticaria/angioedema and in 2 cases anaphylaxis. Analysis of prick tests from 1286 allergy sufferers aged 2-79 years in southern Iran showed positive walnut tests in as many as $21.4 \%$, which was the second most frequent allergy after hen's egg white (30.3\%). Cases of anaphylaxis induced by ACEi after consumption of walnuts were also observed [50, 51].

\section{Summary}

Allergy to nuts (including tree nuts) is an extremely important clinical problem, mainly due to the occurrence of severe reactions caused by the presence in nuts of allergenic granulation proteins from the prolamin and cupin family and non-specific proteins transporting lipids. The presence of so-called latent allergens is also a serious risk since severe reactions can be caused by the trace content of nut allergens present in other foods, which can be found accidentally or can be added as taste enhancers.

The epidemiology of allergy to nuts is also variable and depends mainly on the geographical zone and eating habits. In northern Europe, hazelnut allergy is prevalent, whereas in the USA peanut allergy (and from tree nuts walnut allergy).

Taking into account the above implications and not fully understood composition of allergenic components, also the diagnosis of allergies to nuts is difficult and requires the use of the most modern research tools including molecular diagnostic techniques.

\section{Conflict of interest}

The authors declare no conflict of interest.

\section{References}

1. Szweykowska A, Szweykowski J. Słownik botaniczny. Wiedza Powszechna, Warsaw 2003.

2. Turland N, Wiersema J, Barrie F, et al. International Code of Nomenclature for algae, fungi, and plants (Shenzhen Code) adopted by the Nineteenth International Botanical Congress Shenzhen, China, July 2017. Koeltz Botanical Books ISBN: 978-3-946583-16-5.

3. Bock S, Munoz-Furlong A, Sampson H. Fatalities due to anaphylactic reactions to foods. J Allergy Clin Immunol 2001; 107: 191-3.

4. Sicherer S, Furlong T, Munoz-Furlong A, et al. A voluntary registry for peanut and tree nut allergy: characteristics of the first 5149 registrants. J Allergy Clin Immunol 2001; 108: 128-32.

5. Mandalari G, Mackie A. Almond allergy: an overview on prevalence, thresholds, regulations and allergen detection. Nutrients 2018; 10: 1706-18.

6. Zhang Y, Jin T. Almond allergens: update and perspective on identification and characterization. J Sci Food Agric 2020; 100: 4657-63.

7. Gaur V, Sethi D, Salunke D. Purification, identification and preliminary crystallographic studies of Pru du amandin, an allergenic protein from Prunus dulcis. Acta Crystallogr Sect F Struct Biol Cryst Commun 2008; 64: 32-5.

8. Pitre M, L'Hocine L, Achouri A, et al. Immunoglobulin E-binding pattern of canadian peanut allergic children and crossreactivity with almond, hazelnut and pistachio. Biomolecules 2020; 10: 1091-112.

9. Conti A, Fortunato D, Ortolani C, et al. Determination of the primary structure of two lipid transfer proteins from apricot (Prunus armeniaca). J Chromatogr B Biomed Sci Appl 2001; 756: 123-9.

10. Garcia-Selles F. Patterns of reactivity to lipid transfer proteins of plant foods and Artemisia pollen: an in vivo study. Int Arch Allergy Immunol 2002; 128: 115-22.

11. Uotila R, Kukkonen A, Pelkonen A, et al. Cross-sensitization profiles of edible nuts in a birch-endemic area. Allergy 2016; 71: 514-21.

12. Alcocer M, Rundqvist L, Larsson G. Ber e 1 protein: the versatile major allergen from Brazil nut seeds. Biotechnol Lett 2021; 34: 597-610.

13. Rayes H, Raza A, Williams A, et al. Specific IgE to recombinant protein (Ber e 1) for the diagnosis of Brazil nut allergy. Clin Exp Allergy 2016; 46: 654-6.

14. Beyer K, Bardina L, Grishina G, et al. Identification of sesame seed allergens by 2-dimensional proteomics and Edman sequencing: seed storage proteins as common food allergens. J Allergy Clin Immunol 2002; 110: 154-9.

15. Barre A, Borges JP, Culerrier R, et al. Homology modelling of the major peanut allergen Ara $\mathrm{h} 2$ and surface mapping of IgE-binding epitopes. Immunol Lett 2005; 100: 153-8.

16. Asero R, Mistrello G, Roncarolo D, et al. Walnut-induced anaphylaxis with cross-reactivity to hazelnut and Brazil nut. J Allergy Clin Immunol 2004; 113: 358-60.

17. Arshad S, Malmberg E, Krapf K, et al. Clinical and immunological characteristics of Brazil nut allergy. Clin Exp Allergy 1991; 21: 373-6.

18. Mendes C, Costa J, Vicente, et al. Cashew nut allergy: clinical relevance and allergen characterisation. Clin Rev Allergy Immunol 2019; 57: 1-22.

19. Reitsma M, Bastiaan-Net S, Sforza S, et al. Purification and characterization of Anacardium occidentale (Cashew) aller- 
gens Ana o 1, Ana o 2, and Ana o 3. J Agric Food Chem 2016; 64: 1191-201.

20. Mattison C, Bren-Mattison Y, Vant-Hull B, et al. Heat-induced alterations in cashew allergen solubility and IgE binding. Toxicol Rep 2016; 3: 244-51.

21. Smeekens J, Bagley K, Kulis M. Tree nut allergies: allergen homology, cross-reactivity, and implications for therapy. Clin Exp Allergy 2018; 48: 762-72.

22. Barre A, Sordet C, Culerrier R, et al. Vicilin allergens of peanut and tree nuts (walnut, hazelnut and cashew nut) share structurally related IgE-binding epitopes. Mol Immunol 2008; 45: $1231-40$

23. Costa J, Mafra I, Carrapatoso I, et al. Hazelnut allergens: molecular characterization, detection, and clinical relevance. Crit Rev Food Sci Nutr 2016; 56: 2579-605.

24. Hansen K, Ballmer-Weber B, Sastre J, et al. Componentresolved in vitro diagnosis of hazelnut allergy in Europe. J Allergy Clin Immunol 2009; 123: 1134-41.

25. Jacob T, von Loetzen C, Reuter A, et al. Identification of a natural ligand of the hazel allergen Cor a 1. Sci Rep 2019; 9: 8714-27.

26. Fah J, Wüthrich B, Vieths S. Anaphylactic reaction to lychee fruit: evidence for sensitization to profilin. Clin Exp Allergy 1995; 25: 1018-23.

27. Schocker F, Luttkopf D, Scheurer S, et al. Recombinant lipid transfer protein Cor a 8 from hazelnut: a new tool for in vitro diagnosis of potentially severe hazelnut allergy. J Allergy Clin Immunol 2004; 113: 141-7.

28. Geiselhart S, Hoffmann-Sommergruber K, Bublin M. Tree nut allergens. Mol Immunol 2018; 100: 71-81.

29. Cuadrado C, Sanchiz A, Vicente F, et al. changes induced by pressure processing on immunoreactive proteins of tree nuts. Molecules 2020; 25: 954-66.

30. Rico P, Crespo J, Feliu A, et al. Chestnut allergy: beyond the latex-fruit syndrome. J Allergy Clin Immunol 2004: 113: S149.

31. Lee S, Yoon S, Kim S, et al. Chestnut as a food allergen: identification of major allergens. J Korean Med Sci 2005; 20 : 573-8.

32. Antico A. Oral allergy syndrome induced by chestnut (Castanea sativa). Ann Allergy 1996; 76: 37-40.

33. Herbst R, Wahl R, Frosch P. Specific IgE reactivity and identification of potential allergens in macadamia allergy. J Eur Acad Dermat Venereol 2010; 24: 1361-3.

34. Hansen S. Macadamia nut toxicosis in dogs. Vet Med 2002; 97: 274-6.

35. Yoshida K, Shirane S, Kinoshita K, et al. Macadamia nut allergy in children: clinical features and cross-reactivity with walnut Pediatr Allergy Immunol 2021; 32: 1111-4.

36. Fleischer D, Conover-Walker M, Matsui E, et al. The natural history of tree nut allergy. J Allergy Clin Immunol 2005; 116: 1087-93.

37. De Knop K, Hagendorens M, Bridts C, et al. Macadamia nut allergy: 2 case reports and a review of the literature. Acta Clin Belg 2010; 65: 129-32.

38. Elizur A, Appel M, Nachshon I, et al. Clinical and molecular characterization of walnut and pecan allergy (NUT CRACKER Study). J Allergy Clin Immunol Pract 2020; 8: 157-65.

39. Elizur A, Appel M, Nachshon L, et al. NUT Co Reactivity - ACquiring Knowledge for Elimination Recommendations (NUT CRACKER) study. Allergy 2018; 73: 593-601.

40. Joyce K, Boyd J, Viernes J. Contact dermatitis following sustained exposure to pecans (Carya illinoensis): a case report. Cutis 2006; 77: 209-12.
41. Jacquenet S, Moneret-Vautrin DA. Les allergènes de l'arachide et des fruits à coque. Allergy to peanut and all kinds of nuts Revue francaise d allergologie 2007; 47: 487-91.

42. Costa J, Silva I, Vicente A, et al. Pistachio nut allergy: an updated overview. Crit Rev Food Sci Nutr 2019; 59: 546-62.

43. Rance F, Bidat E, Bourrier T, Sabouraud D. Cashew allergy: observations of 42 children without associated peanut allergy. Allergy 2003; 58: 1311-4.

44. van der Valk J, el Bouche R, van Wijk R, et al. Low percentage of clinically relevant pistachio nut and mango co-sensitisation in cashew nut sensitised children. Clin Transl Allergy 2017; 7: 8-12.

45. Jeong K, Lee S, Ahn K, et al. A multicenter study on anaphylaxis caused by peanut, tree nuts, and seeds in children and adolescents. Allergy 2017; 72: 507-10.

46. Liccardi G, Mistrello G, Noschese P, et al. Oral allergy syndrome (OAS) in pollinosis patients after eating pistachio nuts: two cases with two different patterns of onset. Allergy 1996; 51: 919-22.

47. Pastorello EA, Farioli L, Pravettoni V, et al. Lipid transfer protein and vicilin are important walnut allergens in patients not allergic to pollen. J Allergy Clin Immunol 2004; 114: 908-14.

48. Costa J, Carrapatoso I, Oliveira MB, Mafra I. Walnut allergens: molecular characterization, detection and clinical relevance. Clin Exp Allergy 2014; 44: 319-41.

49. Mew R, Borres M, Sjolander S, et al. A retrospect study into the utility of allergen components in walnut allergy. Pediatr Allergy Immunol 2016; 27: 750-2.

50. Khazaei H, Hashemi S, Aghamohammadi A, et al. The study of type 1 allergy prevalence among people of South-East of iran by skin prick test using common allergens. Iran J Allergy Asthma Immunol 2003; 2: 165-8.

51. Varney V, Nicholas A, Warner A, et al. IgE-mediated systemic anaphylaxis and its association with gene polymorphisms of ACE, angiotensinogen and chymase. J Asthma Allergy 2019; 12: 343-61. 